\title{
Prolonged $\alpha$-amanitin treatment of cells for studying mutated polymerases causes degradation of DSIF160 and other proteins
}

\author{
DAVID C. TSAO, ${ }^{1}$ NOH JIN PARK, ${ }^{1}$ ANITA NAG, ${ }^{2}$ and HAROLD G. MARTINSON ${ }^{3}$ \\ Department of Chemistry and Biochemistry, and the Molecular Biology Institute, University of California at Los Angeles, Los Angeles, California \\ 90095-1569, USA
}

\begin{abstract}
A useful method for studying the function of the mammalian RNA polymerase II takes advantage of the extreme sensitivity of its largest subunit, Rpb1, to $\alpha$-amanitin. Mutations of interest are introduced into an $\alpha$-amanitin-resistant version of Rpb1, which is then expressed ectopically in cells. The phenotypes of these cells are then examined after inhibiting the endogenous wild-type polymerase with $\alpha$-amanitin. Here, we show that cells that are enabled to grow in $\alpha$-amanitin by expression of an $\alpha$-amanitinresistant Rpb1 exhibit changes in cell physiology that can lead to misleading experimental outcomes. The changes we have characterized include the accelerated degradation of some proteins, such as DSIF160, and the reduced rate of synthesis of others. In one series of experiments, we examined an $\alpha$-amanitin-resistant construct, with a mutant C-terminal domain (CTD), that was unable to direct poly(A)-dependent transcription termination in cells growing in $\alpha$-amanitin. The potential interpretation that the termination defect in this construct is due to the mutation in the CTD was rejected when the construct was found to be termination-competent in cells grown in the absence of $\alpha$-amanitin. Instead, it appears that certain termination factors become limiting when the cells are grown in $\alpha$-amanitin, presumably due to the $\alpha$-amanitin-induced degradation we have characterized and/or to the inadequate transcription of certain genes by the $\alpha$-amanitin-resistant Rpb1-containing polymerase.
\end{abstract}

Keywords: $\alpha$-amanitin; cleavage and polyadenylation; poly(A)-dependent termination; RNA polymerase II; CTD; DSIF

\section{INTRODUCTION}

Genetic analysis of RNA polymerase II (Pol II) function in mammalian cells is facilitated, in the case of its largest subunit Rpb1, by the extreme sensitivity of Rpb1 to inhibition by $\alpha$-amanitin ( $\alpha$-ama). In such experiments, $\alpha$-ama-resistant Rpb1 subunits $\left(\mathrm{Rpb} 1_{\mathrm{r}}\right)$, bearing mutations of interest, are ectopically expressed in cells treated with $\alpha$-ama. The $\alpha$-ama prevents transcription by any Pol II that contains the wild-type Rpbl so that one can monitor, unambiguously, the activities of the mutant (Bartolomei et al. 1988; Meininghaus et al. 2000; Fong

${ }^{1}$ Present address: Department of Molecular Genetics, Quest Diagnostics Nichols Institute, 33608 Ortega Highway, San Juan Capistrano, CA 92690, USA.

${ }^{2}$ Present address: Department of Biological Science, Florida State University, Tallahassee, FL 32306, USA.

${ }^{3}$ Corresponding author.

E-mail hgm@chem.ucla.edu.

Article published online ahead of print. Article and publication date are at http://www.rnajournal.org/cgi/doi/10.1261/rna.030452.111. and Bentley 2001; Laurencikiene et al. 2006; Martins et al. 2011; Sims et al. 2011).

One of the most extensively studied regions of Rpb1 is its C-terminal domain (CTD). This is an essential and highly unusual domain, often simply called the Pol II CTD. In vertebrates, the CTD consists of 52 tandemly repeated heptapeptides terminated by an additional 10-amino acid sequence. One of the principal roles of the CTD appears to be the orchestration of diverse interactions with a multitude of proteins that modulate Pol II function and that serve to integrate transcription with numerous downstream processes, such as capping, splicing, and cleavage/polyadenylation of the transcript (Buratowski 2009).

The fact that transcription is integrated with downstream events was first demonstrated by the finding that termination of transcription at protein-coding genes in mammalian cells depends on the poly(A) signal, an RNA motif required for 3 '-end processing of the transcript (Whitelaw and Proudfoot 1986). Subsequent work has established a strong correlation between the molecular requirements for poly(A)dependent termination and for cleavage/polyadenylation, 
both of which rely on the poly(A) signal (Richard and Manley 2009; Kuehner et al. 2011). However, it remains unclear whether one process actually depends on the other or whether the two processes are driven independently by the same bifunctional apparatus (Qu et al. 2006).

The studies described in the present communication were motivated by previous reports that a particular $R p b 1_{r}$, whose CTD lacks the final 27 heptads as well as the terminal 10 amino acids, was unimpaired in its ability to support poly(A)-dependent termination (Park et al. 2004), yet was deficient in yielding transcripts that were successfully processed at the $3^{\prime}$ end (Fong and Bentley 2001; Fong et al. 2003). One possible interpretation of these results is that poly $(\mathrm{A})$-dependent termination can proceed independently of poly(A) site cleavage of the transcript. An alternative, but not mutually exclusive, interpretation is that the cleavage and polyadenylation deficiency in the experiments of Fong and colleagues resulted from treating the cells with $\alpha$-ama. Cells were not treated with $\alpha$-ama in the termination experiments of Park and colleagues. Instead, nuclei were isolated from healthy cells, and $\alpha$-ama was used only for the termination assay, carried out on the isolated nuclei by runon transcription.

To determine whether the treatment of cells with $\alpha$-ama may give rise to indirect effects relevant to transcription and its downstream events, we investigated the effects of $\alpha$-ama on transcription termination within cells and its effects on the cellular levels of some representative proteins related to transcription and processing. The results show that, under certain conditions, $\alpha$-ama treatment of cells leads to the selective degradation of DSIF160 and other proteins and interferes with the ability of even $\alpha$-amaresistant polymerases to interact functionally with the poly(A) signal.

\section{RESULTS}

\section{An Rpb1 $1_{r}$ with partially truncated CTD can support transcription termination in healthy cells but not in cells treated with $\alpha$-amanitin}

The rationale for studying CTD mutations by using ectopically expressed $R p b 1_{r}$ as a vehicle is that this allows the inhibition, using $\alpha$-ama, of any Pol II that contains the coexpressed endogenous wild-type Rpb1. For transcription termination assays, the procedure is simple-nuclei are isolated from cells producing both the ectopically expressed $R \mathrm{pb} 1_{\mathrm{r}}$ as well as the wild-type Rpb1, and then nuclear runon transcription is carried out in the presence of $\alpha$-ama. Only the $\mathrm{Rpb} 1_{\mathrm{r}}$-containing Pol II $\left(\mathrm{PolII}_{\mathrm{r}}\right)$ will yield a signal under these conditions, and the ability of the PolII $I_{r}$ to terminate can be inferred from its presence or absence downstream from termination elements. For the assessment of processing, however, the RNA to be characterized must be produced in vivo. This requires that the cells be able to survive growth in $\alpha$-ama long enough for the PolII $\mathrm{r}_{\mathrm{r}}$ to produce and support the processing of sufficient RNA to be assayed (and also to allow all RNA previously synthesized by the wild-type Pol II to exit the nucleus). This extended growth in $\alpha$-ama, in turn, requires that the PollI ${ }_{r}$ (notwithstanding any deficiencies in its mutant CTD) be able to support the transcription and processing required for maintenance of an RNA-processing infrastructure within the cell. Moreover, in the presence of $\alpha$-ama (and an $R p b 1_{r}$ ), synthesis and degradation of the wild-type Rpbl presumably occurs continuously (Nguyen et al. 1996; Anindya et al. 2007). Therefore, the possibility, in such experiments, that this influence of $\alpha$-ama on cell physiology may indirectly affect pre-mRNA processing must be considered.

To determine whether the growth of cells in $\alpha$-ama might affect the ability of a PolII $\mathrm{r}_{\mathrm{r}}$ to engage in post-transcriptional activities, we set out to assay poly(A)-dependent termination in the nuclei of Rpb1-transfected cells that either had or had not been grown for a period of time in $\alpha$-ama. The reporter construct used for these experiments is shown in Figure 1A. Figure $1 \mathrm{~B}$ shows a typical termination assay for cells not grown in $\alpha$-ama but expressing an $R p b 1_{r}$. Following transfection of both the Rpb1 $1_{\mathrm{r}}$ expression plasmid and the reporter into cells, nuclei were isolated, and termination was assayed by run-on transcription in the presence of $\alpha$-ama (Nag et al. 2007). Termination of PolII $\mathrm{r}_{\mathrm{r}}$ transcription downstream from the poly $(\mathrm{A})$ site is indicated by the reduced 174-nt G-less cassette signal compared with that of the 136-nt G-less cassette (Fig. 1B, lane 1). The endogenous $\alpha$-ama-sensitive Pol II can be seen to behave similarly when assays are conducted in the absence of $\alpha$-ama on nuclei from cells lacking any transfected $\mathrm{Rpb}_{\mathrm{r}}$ (last lane of Fig. 5B in Orozco et al. [2002]). Figure 1B, lane 2, shows that poly (A)-dependent termination does not occur downstream from a mutant poly(A) signal.

Figure $1 \mathrm{~B}$, lane 3 , illustrates the use of this assay to characterize a CTD mutation, in this case, the deletion of the entire CTD (Park et al. 2004). When the PolII $r$ lacks its CTD, the 174-nt cassette signal is not diminished in intensity downstream from a poly(A) signal, showing that the CTD is required for termination. The lack of termination is especially obvious when the reduced efficiency of transcription initiation by the CTD-less PolII ${ }_{\mathrm{r}}$ (Gerber et al. 1995) is taken into account (Fig. 1B; cf. the 136-nt cassette signals in lanes 3 and 4 and those in lanes 1 and 2). Indeed, when compared to the 136-nt cassette signal, the 174-nt cassette signal is actually much stronger than expected from mere lack of termination (cf. lane 3 and lane 2). This is thought to reflect the slowing of transcription (and thus the increase in occupancy of polymerases on the template) that normally precedes termination (Orozco et al. 2002; Nag et al. 2007) but which continues indefinitely when termination is abrogated (Park et al. 2004). A template with a mutant poly(A) signal does not induce slowing of transcription by the CTD-less PolII ${ }_{\mathrm{r}}$ (Fig. 1B, lane 4). 
Figure 1C shows the application of the assay illustrated in Figure $1 \mathrm{~B}$ to the $\mathrm{Rpb}_{\mathrm{r}}$ mentioned in the Introduction, which has a partially deleted CTD. Recall that this $R p b 1_{r}$ supports transcription termination in run-on assays carried out on nuclei isolated from healthy cells (Park et al. 2004) but is deficient in supporting the production of processed RNA in cells that had been treated with $\alpha$-ama (Fong and Bentley 2001; Fong et al. 2003). To determine whether $\alpha$-ama treatment of cells can compromise the ability of this PolII $_{r}$ to respond to the poly $(\mathrm{A})$ signal, we carried out poly(A)-dependent termination assays on nuclei isolated from healthy cells or from cells exposed to $\alpha$-ama for various lengths of time (Fig. 1C). For cells not exposed to $\alpha$-ama, poly (A)-dependent termination occurred normally for the reporter with a wild-type poly(A) signal (cf. lane 2 and lane 1). However, by the time the cells had been in $\alpha$-ama for $5.5 \mathrm{~h}$, the termination activity was gone (lane 5 , note the increase in post-cassette signal compared with lane 2 , despite the decrease in pre-cassette signal owing to reduced overall transcription). Several such experiments are summarized in the 1-25 CTD curve of Figure 1D, which traces the increase in post-cassette/pre-cassette ratio (i.e., the loss in termination activity) as a function of the time spent by the cells in $\alpha$-ama prior to the termination assay. Additional experiments show that the poly(A) signaldependent slowing of transcription displayed by the CTD-less PolII $\mathrm{r}_{\mathrm{r}}$ of Figure $1 \mathrm{~B}$, lane 3, was also affected by growing the cells in $\alpha$-ama, but in this case, the effect was augmented ("No CTD" curve) (Fig. 1D). In contrast, the behavior of two other CTD constructs appeared to be unaffected by growing the cells in $\alpha$-ama (27-52 CTD and Full CTD curves) (Fig. 1D).

The loss in ability to terminate by the

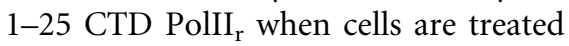
with $\alpha$-ama (Fig. 1C,D) is reminiscent of its inability to support the production of processed RNA in cells growing in $\alpha$-ama (Fong and Bentley 2001; Fong et al. 2003). To check this correlation for the experiment of Figure 1C, we set aside a portion of each nuclei preparation for a parallel RNase protection assay (Fig. 1E). The results confirmed that the production of $3^{\prime}$ processed RNA in cells does, indeed, diminish as a function of time spent by the cells in $\alpha$-ama. Note that the loss in ability to produce processed RNA in Figure 1E, lane 6 , does not necessarily mean that this truncated CTD cannot support processing-just as the failure of poly(A)-dependent termination in lanes 5 and 6 of Figure $1 \mathrm{C}$ does not mean that this truncated CTD cannot support poly(A)-dependent termination (which it does in lane 2 of Fig. 1C).

The experiments described so far have involved the SV40 early poly(A) signal. To determine whether treatment of cells with $\alpha$-ama would compromise the ability of another poly $(A)$ signal to drive termination, we repeated some of the above experiments with the SV40 late poly(A) signal, which differs considerably in sequence from the SV40 early signal and is about five times stronger (Carswell and Alwine 1989; Orozco et al. 2002). Figure 1F shows that, as for the SV40 early poly(A) signal, transcription termination by the

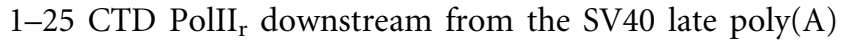
signal, diminishes when the cells are exposed to $\alpha$-ama. However, the negative effect of $\alpha$-ama on the SV40 late poly(A) signal (1-25 CTD curve in Fig. 1F) is somewhat less than on the SV40 early signal (1-25 CTD curve in Fig.
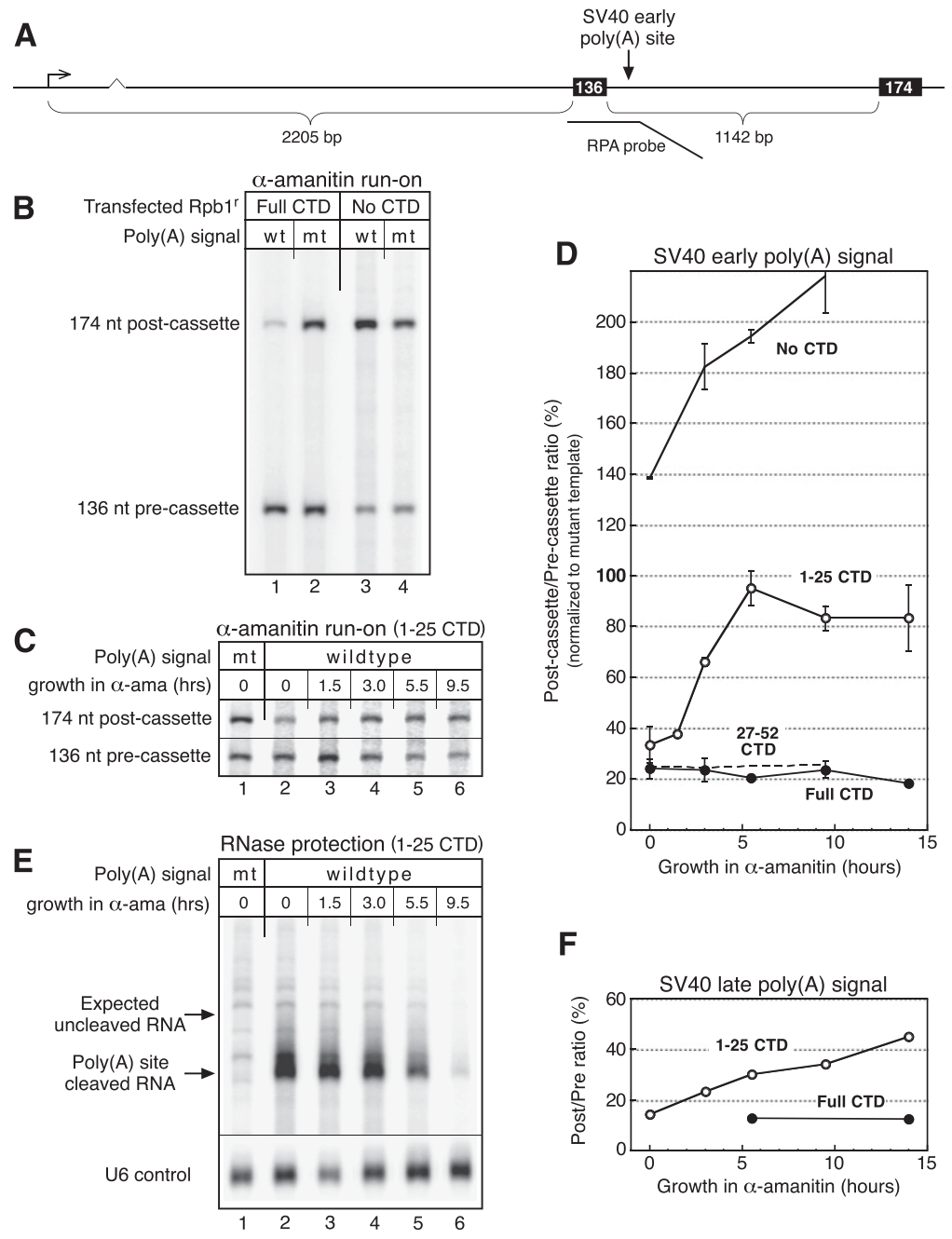

FIGURE 1. (Legend on next page) 
1D). This is consistent with the possibility that $\alpha$-ama decreases the concentration in the cell of one or more factors required for termination and that the stronger SV40 late $\operatorname{poly}(\mathrm{A})$ signal is more capable of recruiting these factors at that lower concentration.

\section{Treatment of cells with $\alpha$-amanitin causes selective protein degradation within the cell}

It is well-known that the wild-type $\alpha$-ama-sensitive Rpb1 is degraded upon treatment of cells with $\alpha$-ama (Guialis et al. 1979; Nguyen et al. 1996; Anindya et al. 2007). However, the results of Figure 1 suggest that other proteins may be affected as well, even if the wild-type Rpb1 is ectopically replaced with an $\alpha$-ama-resistant version. To search for other proteins that might be lost upon $\alpha$-ama treatment, we used Western blots to examine the protein content of cells that had or had not been exposed to $\alpha$-ama. After blotting for just a few proteins, we observed dramatic losses for two of them, DSIF160 and cyclin F. Figure 2A shows that after $14 \mathrm{~h}$ in $\alpha$-ama, cells expressing the 1-25 CTD $\mathrm{Rpb}_{\mathrm{r}}$ (lanes 1-6) have lost almost their entire inventory of these two proteins.

Initially, we had expected to observe losses of one or more of the well-known cleavage/polyadenylation factors, such as CstF. However, Figure 2A shows that the effect of $\alpha$-ama on CstF64 levels is relatively minor, especially when compared to the presumptive loading control in this experiment, TBP. To determine whether these reduced levels of CstF might be sufficient to affect transcription termination, we used RNAi. Figure $2 \mathrm{~B}$ shows that the RNAi-mediated knockdown of CstF to levels that are less than those observed during $\alpha$-ama treatment of cells has no deleterious effect on transcription termination (the post/ pre ratio remains low even after knockdown). A similar experiment in which both CstF64 and CstF77 were knocked down gave the same result (data not shown). In contrast, transcription termination is completely abolished by elimination of the G/U-rich region in the RNA, to which CstF64 binds (Nag et al. 2006). Thus, although CstF64 is presumably required for termination in mammalian cells, as it is in yeast (Birse et al. 1998; Aranda and Proudfoot 2001), the amount of CstF64 that remains after knockdown is still sufficient to fulfill this function. This indicates that the relatively small decrease in CstF caused by $\alpha$-ama (Fig. 2A) is not, at least by itself, responsible for the $\alpha$-ama-induced loss in ability of the 1-25 CTD PolII $r$ to terminate transcription (Fig. 1C,D). Nevertheless, the loss of cleavage/polyadenylation-related functions upon incubation of cells in $\alpha$-ama (Fig. 1C,E) suggests that, directly or indirectly, there is some cleavage/polyadenylation factor(s) that is susceptible to $\alpha$-ama-induced loss of function.

To learn more about the $\alpha$-ama effect, we decided to focus on DSIF160. Figure 2C shows that both high and low concentrations of $\alpha$-ama induce loss of DSIF160. Figure 2D, lanes 6 and 8, show that the 27-52 CTD and Full CTD Rpb1 $1_{r}$ constructs, though able to rescue transcription termination (Fig. 1D) and pre-mRNA processing (Fong and Bentley 2001; Fong et al. 2003) from the effects of $\alpha$-ama, cannot efficiently rescue DSIF160 from $\alpha$-ama-induced degradation. Thus, the effects of $\alpha$-ama are complex, with different functions being affected in different ways. This conclusion is reinforced by the results of Figure 2E, which show again, using a different $\mathrm{Rpb} 1_{\mathrm{r}}$ construct, that $\alpha$-ama causes DSIF160 to be lost under conditions where the ability of transcription to terminate is rescued.

The loss of DSIF160 in the presence of $\alpha$-ama could reflect impaired DSIF160 synthesis coupled with a short half-life, or it could reflect accelerated degradation. Figure $3 \mathrm{~A}$ shows that blocking protein synthesis with cycloheximide 
A
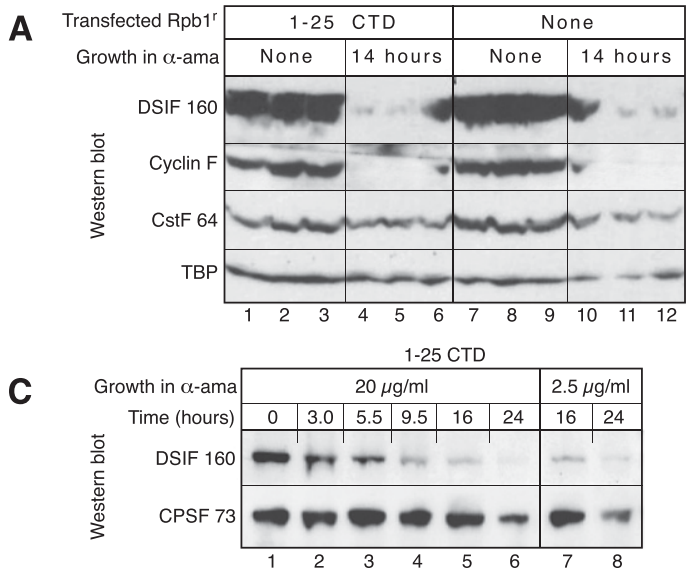

\begin{tabular}{|l|l|l|l|l|l|}
\cline { 2 - 6 } & No CTD & 1-25 CTD & 27-52 CTD & Full CTD & NE \\
\hline
\end{tabular}

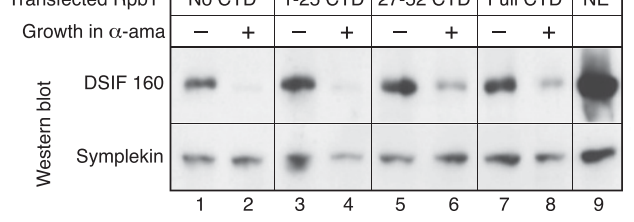

FIGURE 2. Treatment of cells with $\alpha$-amanitin causes selective protein loss within the cell. $(A)$ DSIF160 and cyclin F are lost when cells expressing the 1-25 CTD Rpb $1_{\mathrm{r}}$ are incubated in $\alpha$-ama. Nuclei were prepared in triplicate from cell cultures essentially equivalent to those used for the zero- and 14-h time points of the 1-25 CTD curve of Figure 1D. Nuclear proteins were blotted for DSIF160, CstF64, TBP and, after stripping, cyclin F. Note that there is considerable spillover from lanes 7 and 9 into lanes 6 and 10, which is most noticeable for DSIF and cyclin F. (B) Moderate RNAi-induced knockdown of CstF64 has no effect on transcription termination. Transfection of 293T cells with siRNA (Dharmacon SMARTpool M-011245-00) was carried out based on the procedure of Wagner and Garcia-Blanco (2002), with the siRNA transfections on days 2, 4, and 6 (all using Lipofectamine 2000; Invitrogen) and the reporter transfection on day 5 (using Fugene 6; Roche). The "\% CstF64 remaining" in lanes 3 and 4 was calculated relative to the mock transfections of lanes 1 and 2 , after normalizing to the amount of TBP present. The "Post/Pre ratio" was calculated as for Figure 1D,F. (C) DSIF160 is subject to $\alpha$-ama-induced loss at both high and low concentrations of $\alpha$-ama. $(D) \alpha$-ama causes loss of DSIF160 regardless of whether there is also an effect on RNA processing or transcription termination. Cells for the "+" lanes were grown in $2.5 \mu \mathrm{g} / \mathrm{mL}$ of $\alpha$-ama for $24 \mathrm{~h}$. (E) Confirmation, using the 1-22 CTD Rpb1 $1_{\mathrm{r}}$ expression construct (Rosonina and Blencowe 2004), that DSIF160 is more susceptible to $\alpha$-ama-induced loss (Western blot) than are termination factors (Run-on assay). The $\alpha$-ama, dissolved in DMSO, was added to a concentration of $20 \mu \mathrm{g} / \mathrm{mL}$ for the 14-h time point, and DMSO alone was added for the no $\alpha$-ama control. The run-on assay panel presents the average and standard deviation for three independent experiments conducted in 6-well plates. The Western blot shows the results of a single scaled-up experiment using $60-\mathrm{mm}$ plates.

(Hinkson and Elias 2011) does not result in rapid loss of DSIF160. Indeed, the half-life of DSIF160 appears to be comparable to that of CstF64 and HDAC-1 (chosen arbitrarily for comparison) and much greater than that of cyclin F, a protein of known short half-life (Fung et al. 2002). This suggests that the $\alpha$-ama-induced loss of DSIF160 reflects accelerated DSIF160 degradation. To test this possibility, we treated cells with $\alpha$-ama in the presence or absence of MG132, a proteasome inhibitor (Lee and Goldberg 1998). Figure 3B shows that MG132 rescued DSIF160 from $\alpha$-ama-induced loss (cf. lanes 3 and 6 with lanes 2 and 5 , respectively). Cyclin $\mathrm{F}$ was also rescued from $\alpha$-amainduced loss by MG132, albeit in a form with reduced mobility on the gel (Cope and Deshaies [2006] have also observed a slow form of cyclin $\mathrm{F}$ in MG132). In contrast, the amount of CstF64 in $\alpha$-ama-treated cells was not increased at all by the presence of MG132 (cf. lanes 3 and 6 with lanes 2 and 5 , respectively). The effect of MG132 on the amount of HDAC-1 in $\alpha$-ama-treated cells was similarly small.

We wondered whether the addition of MG132 would rescue the ability of the 1-25 CTD Rpb1 $1_{\mathrm{r}}$ to support transcription termination in $\alpha$-ama-treated cells. Recall that this particular Rpb1 $1_{r}$ is unable to support transcription termination downstream from the SV40 early poly(A) signal when ectopically expressed in cells that are treated with $\alpha$-ama (Fig. 1C,D). Figure 3C shows that MG132 partially restores the ability to terminate under these circumstances, suggesting that some, but not all, of the functional deficit caused by $\alpha$-ama is due to protein degradation in the cells.

It is interesting that the ectopic expression of certain $\mathrm{Rpb} 1_{\mathrm{r}}$ constructs can fully rescue transcription termination in $\alpha$-ama-treated cells (Figs. 1D, 2E) despite the continued degradation of DSIF160 (Fig. 2D,E). This suggests that it is not the ability of MG132 to rescue DSIF160 from degradation (Fig. 3B) that partially restores transcription termination to $\alpha$-ama-treated cells (Fig. 3C). Thus, we can identify at least three groups of proteins whose presence or function is impacted by the presence of $\alpha$-ama in cells-DSIF160, cyclin F, and, presumably, other proteins that are degraded but not required for transcription termination; unknown proteins, required for termination, that can be rescued by MG132 in cells expressing the 1-25 CTD Rpb1 $1_{r}$ (Fig. 3C); and other unknown proteins important for termination that cannot be rescued by MG132 (Fig. 3C).

\section{Inadequate Rpb1 $\mathbf{r}_{\mathrm{r}}$ expression in $\alpha$-ama-treated cells allows transcription initiation but not termination}

We have seen (Fig. 1C) that the 1-25 CTD Rpb1 $1_{\mathrm{r}}$ is able to support transcription termination within cells that also express normal levels of the wild-type Rpb1 (i.e., in cells not exposed to $\alpha$-ama) but not in cells that depend exclusively on the $1-25 \mathrm{Rpb}_{\mathrm{r}}$ for Pol II activity (i.e., in cells growing in $\alpha$-ama). In contrast, the $27-52 \mathrm{CTD}$, the Full CTD, and the 1-22 CTD Rpb1 $1_{\mathrm{r}}$ constructs can support 
A

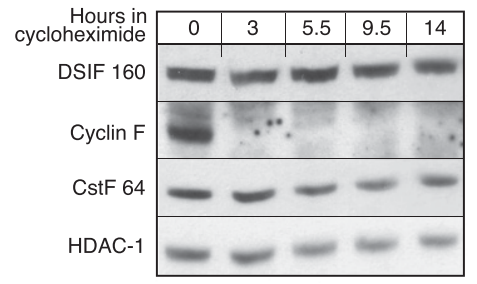

B

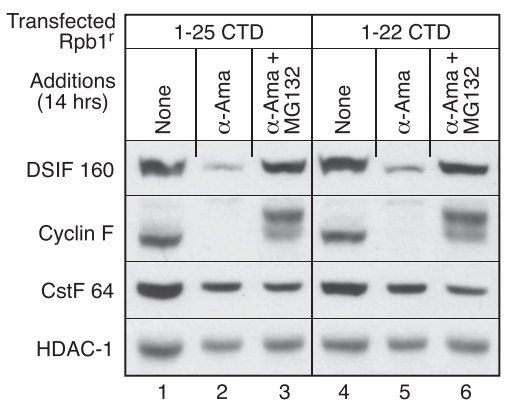

C

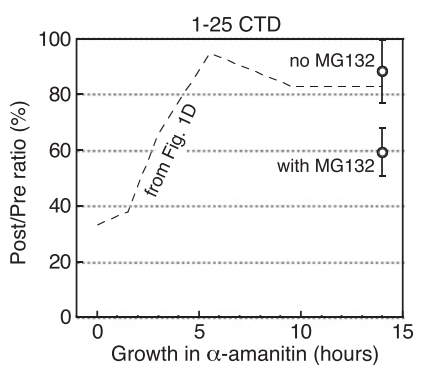

FIGURE 3. $\alpha$-amanitin induces protein degradation. (A) DSIF160 does not normally have a short half-life. Untransfected cells were cultured for three days in 35-mm plates, and then nuclei were isolated and assayed by Western blotting. At the indicated number of hours before harvest, cycloheximide was added to a final concentration of 20 $\mu \mathrm{g} / \mathrm{mL}$. (B) Inhibition of protein degradation prevents the $\alpha$-amainduced loss of DSIF160. The data were obtained essentially as described for the Western blot of Figure 2D. For lanes 3 and 6, MG132, at a final concentration of $10 \mu \mathrm{M}$, was included with the $\alpha$-ama. (C) Inhibition of protein degradation partially rescues $\alpha$-amainhibited transcription termination. Cells treated with $20 \mu \mathrm{g} / \mathrm{mL}$ of $\alpha$-ama for $14 \mathrm{~h}$ were grown in parallel with cells treated simultaneously with $\alpha$-ama and $10 \mu \mathrm{M}$ MG132, and assayed for termination in a manner similar to that described for Figure 1C. The results shown are the average and standard deviation from five independent experiments. Shown for comparison is the 1-25 CTD curve from Figure 1D. In fact, the "no MG132" value comes from a subset of the 14-h time point samples of the 1-25 CTD curve.

transcription termination even when they are the sole source of Rpb1 activity (Figs. 1D, 2E). This suggests that, although these three constructs cannot prevent the $\alpha$-amainduced degradation of certain proteins such as DSIF160 (Figs. 2D,E, 3B), they, nevertheless, do support the production of sufficient levels of other (as yet unidentified) proteins required for transcription termination. A significant distinction between the 1-25 CTD Rpb $1_{\mathrm{r}}$ and the other $R p b 1_{r}$ constructs is that the $1-25 R p b 1_{r}$ is expressed poorly, possibly reflecting a destabilizing effect of that particular CTD mutation on the Rpb1 (Chapman et al. 2005), but the others are expressed well, in transfection experiments (Park et al. 2004; Rosonina \& Blencowe 2004). Therefore, we wondered whether the ability of the 27-52 CTD, the Full CTD, and the 1-22 CTD Rpb $1_{\mathrm{r}}$ constructs to support termination, when they are the sole source of Rpb1 activity in the cell, might be related to the robustness of their expression following transfection.

To determine whether the Rpb1 $1_{\mathrm{r}}$ expression level would affect the efficiency of transcription termination, we carried out the experiment of Figure 4. Lanes 1 and 2 show that, when the 1-25 CTD and 1-22 CTD Rpb1 $1_{\mathrm{r}}$ constructs were each coexpressed with the endogenous wild-type Rpb1 (i.e., cells grown in the absence of $\alpha$-ama), they supported transcription termination downstream from the SV40 early poly(A) signal with similar efficiencies, in agreement with Figures $1 \mathrm{D}$ and $2 \mathrm{E}$. Also in agreement with the previous figures, when expression of the endogenous Rpb1 was prevented with $\alpha$-ama, termination by the $1-25 \mathrm{Rpb} 1_{\mathrm{r}}$ (lane 3), but not by the 1-22 Rpb1 $1_{\mathrm{r}}$ (lane 4), was inhibited. However, when the amount of transfecting 1$22 \mathrm{Rpb} 1_{\mathrm{r}}$ expression plasmid was gradually reduced (lanes 5-8), the efficiency of termination was similarly diminished, ultimately approaching the level of the poorly expressed 1-25 Rpb1 $1_{\mathrm{r}}$ (lane 3). The simplest interpretation of these results is that inadequate amounts of one or more short-lived proteins, required for termination, are produced when very small amounts of expression plasmid are transfected. Note, however, that even when there is insufficient $\mathrm{Rpb} 1_{\mathrm{r}}$ to produce essential proteins for transcription termination, the factors required for transcription itself (i.e., the polymerase and its initiation and elongation factors) remain at sufficient levels to produce

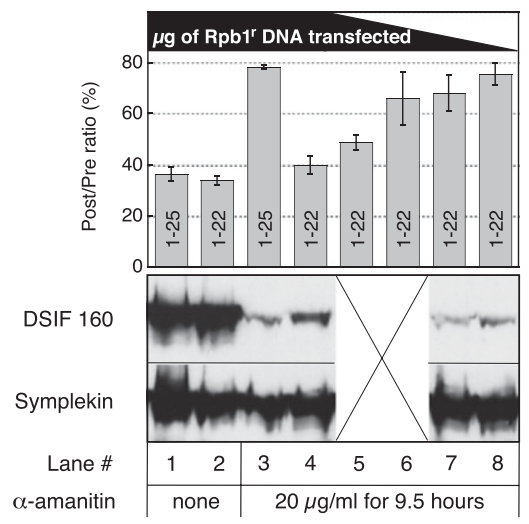

FIGURE 4. Maintenance of adequate levels of some termination factors depends on the expression of sufficient Rpb1. Lanes 1-4 show a typical experiment in which cells in $35-\mathrm{mm}$ plates were transfected with $0.1 \mu \mathrm{g}$ of either the 1-25 CTD or the 1-22 CTD Rpb1 $1_{\mathrm{r}}$ construct. After growing without or with $\alpha$-ama for $9.5 \mathrm{~h}$, each sample was split for run-on and Western blot assays. Lanes 5-8 show the results of forcing the cells to survive on progressively less transfected 1-22 $\mathrm{Rpb} 1_{\mathrm{r}}$, in which the amount of $1-22 \mathrm{Rpb} 1_{\mathrm{r}}$ DNA transfected was $4 \%$, $2 \%, 1 \%$, and $0.5 \%$ as much as that for lanes $1-4$. No Western blot was carried out for lanes 5 and 6 . 
the nonterminating read-through transcription that is detected in our assays (e.g., Fig. 1C, lanes 5 and 6).

\section{DISCUSSION}

Mammalian Pol II Rpb1 function can be studied by use of elegant genetic experiments in which the endogenous polymerase is inactivated with $\alpha$-ama while a mutant Rpb1 is provided ectopically in an $\alpha$-ama-resistant form. However, this approach usually requires growing cells in the presence of $\alpha$-ama, which raises the possibility that offtarget or indirect effects of $\alpha$-ama on cell physiology may influence phenotype in ways that need to be distinguished from any direct effects that the Rpb1 mutation may have on function. For example, the 1-25 CTD Rpb $1_{\mathrm{r}}$ used in Figure $1 \mathrm{C}$ can initiate and elongate transcription in cells treated with $\alpha$-ama but exhibits a deficit in termination of transcription (lanes 5 and 6). Yet, this defect is not a direct consequence of the CTD mutation on termination, because the 1-25 CTD Rpb1 $1_{\mathrm{r}}$ can support transcription termination just fine within cells not growing in $\alpha$-ama (Fig. 1C, lane 2 ). Instead, the termination defect is an indirect consequence of the fact that cells growing in $\alpha$-ama are forced to rely on the poorly expressed 1-25 CTD Rpb1 $1_{\mathrm{r}}$ polymerase for all of their Pol II transcription, which apparently results in the insufficient production of unidentified short half-life proteins required for termination (Fig. 4). Consistent with this possibility, inhibiting protein degradation while cells are grown in $\alpha$-ama partially restores transcription termination by the 1-25 CTD construct (Fig. 3C).

Growing cells in $\alpha$-ama also leads directly to protein degradation, even with $R \mathrm{pb} 1_{\mathrm{r}}$ constructs that can support unimpaired transcription termination in the $\alpha$-ama-grown cells (e.g., Fig. 2D, lanes 6 and 8; Fig. 2E). The phenomenon of protein degradation in $\alpha$-ama-treated cells is wellknown. Blocking transcription with $\alpha$-ama leads to stalling of transcription complexes and wholesale degradation of the large subunit of the endogenous polymerase (Nguyen et al. 1996; Anindya et al. 2007). Moreover, this process is presumably ongoing in $\mathrm{Rpb}_{\mathrm{r}}$-rescued cells growing in the presence of $\alpha$-ama because the $\alpha$-ama does not block synthesis of the wild-type Rpb1. It is possible that these widespread upheavals in the cell have significant ripple effects on other processes. Ironically, despite the multiple effects of $\alpha$-ama documented here, degradation of Rpb1 in the presence of $\alpha$-ama is actually quite specific - it appears to be a consequence of the blockage of transcription per se (Anindya et al. 2007) and it is not perceptibly accompanied by the degradation of certain other subunits of the polymerase itself (Nguyen et al. 1996). It is, perhaps, for this reason that the possibility of wide-ranging indirect effects of $\alpha$-ama on cell physiology has not previously received close scrutiny in experiments involving the use of $\alpha$-ama.

When we began these experiments, we searched for effects on the major cleavage and polyadenylation proteins because these are known to be involved in poly(A)dependent termination. Although we included DSIF160 and cyclin F only as controls, it is they, not the polyadenylation proteins, that are lost when cells are exposed to $\alpha$-ama (Fig. 2). The loss of cyclin $F$ in $\alpha$-ama grown cells (Fig. 2A) may be related to its short half- life (Fig. 3A), but we note that this loss is not rescued by the 1-22 CTD Rpb $1_{\mathrm{r}}$ (Fig. 3B, lane $5)$, which rescues completely the ability to terminate transcription (Fig. 2E). In contrast to cyclin F, DSIF160 does not have a particularly short half-life (Fig. 3A), so its loss is unambiguously due to $\alpha$-ama-induced degradation, consistent with the observation that loss of DSIF160 is prevented by blocking protein degradation with MG132 (Fig. 3B). Regarding the $3^{\prime}$-end processing proteins, there does appear to be some loss of CPSF after long times in $\alpha$-ama (Fig. 2C), but the rate of CPSF loss is much lower than the rate at which the ability to terminate transcription is lost (Fig. 1C,D). Similarly, although there is often some loss of CstF when cells are treated with $\alpha$-ama (Figs. 2A,E, 3B), greater losses due to RNAi have no effect on termination (Fig. 2B).

We have not found conditions for which an ectopically expressed $R p b 1_{\mathrm{r}}$ could entirely eliminate the physiological perturbations of growing cells in $\alpha$-ama. Even expressing an $R \mathrm{Rb} 1_{\mathrm{r}}$ with a completely intact CTD did not rescue DSIF160 from degradation (Fig. 2D, lane 8), despite the fact that this $\mathrm{Rpb}_{\mathrm{r}}$ can fully support efficient transcription termination (Fig. 1D). It is possible that certain Rpb1-interacting proteins, of which DSIF160 (Yamaguchi et al. 1999) and Rpb2 (Guialis et al. 1979) would be examples, are at risk for continuous codegradation (Papavassiliou et al. 1992) in $\alpha$-ama-grown cells, along with the continuously synthesized and degraded endogenous Rpb1. And this may be what is responsible for the month-long crisis that longterm cell cultures go through (despite stably expressing a fully intact $R p b 1_{\mathrm{r}}$ ) as they adapt to growth in $\alpha$-ama (Meininghaus et al. 2000). Finally, we note that codegradation of Rpb1-interacting elongation factors would be of particular relevance to experiments that examine parameters and processes related to transcription elongation in cells growing in $\alpha$-ama.

\section{MATERIALS AND METHODS}

All Rpb1 $1_{\mathrm{r}}$ expression plasmids were provided by David Bentley (Fong and Bentley 2001), except for the 1-22 CTD Rpb $1_{\mathrm{r}}$, which was from Ben Blencowe (Rosonina and Blencowe 2004). Run-on transcription was carried out on COS cell nuclei that were lysed in high salt and heparin to dissociate most proteins from the DNA and its engaged polymerases (Park et al. 2004; Nag et al. 2007). Addition of $\alpha$-ama to the run-on reaction $(0.3 \mu \mathrm{g} / \mathrm{mL}$ final concentration) ensured that only the ectopically expressed Rpb $1_{r}$ were detected. The distance transcribed during run-on is short so the amount of $\left[{ }^{32} \mathrm{P}\right]$ RNA produced is essentially proportional to the number of polymerases engaged on the template. Selected regions of the template code for G-less cassettes. When isolated by $\mathrm{T} 1 \mathrm{RNase}$ digestion, the intensity of $\left[{ }^{32} \mathrm{P}\right]$ label in the cassettes 
of various lengths is proportional to the number of polymerases within the cassette boundaries on the template. When living cells were treated with $\alpha$-ama, higher concentrations were used than for run-on transcription to ensure rapid entry into the cell (Nguyen et al. 1996). For experiments presented here, two different concentrations ( 2.5 or $20 \mu \mathrm{g} / \mathrm{mL}$ ) were used, which we have found to be essentially interchangeable.

Western blotting was carried out according to the BM Chemiluminescence Western Blotting Kit (Roche) protocol, on nuclei prepared as previously described (Park et al. 2004). Nitrocellulose membranes were used for protein transfer and, for stripping, the Restore Plus Western Blot stripping buffer (Thermo) was used. Anti-B10 was from Chemicon; anti-DSIF160 and anti-symplekin were from BD Signal Transduction; and anti-TBP (N12), anticyclin F (C20), and anti-HDAC-1 (H51) were from Santa Cruz Biotechnology. Anti-CPSF73 and anti-CstF64 (3A7) were gifts from David Bentley and Clint MacDonald, respectively.

\section{ACKNOWLEDGMENTS}

We thank David Bentley (University of Colorado) and Clint MacDonald (Texas Tech) for antibodies, and Benson Ngo and Huimin Zhang for comments on the manuscript.

Received September 20, 2011; accepted November 4, 2011.

\section{REFERENCES}

Anindya R, Aygun O, Svejstrup JQ. 2007. Damage-induced ubiquitylation of human RNA polymerase II by the ubiquitin ligase Nedd4, but not Cockayne syndrome proteins or BRCA1. Mol Cell 28: $386-397$.

Aranda A, Proudfoot N. 2001. Transcriptional termination factors for RNA polymerase II in yeast. Mol Cell 7: 1003-1011.

Bartolomei MS, Halden NF, Cullen CR, Corden JL. 1988. Genetic analysis of the repetitive carboxyl-terminal domain of the largest subunit of mouse RNA polymerase II. Mol Cell Biol 8: 330-339.

Birse CE, Minvielle-Sebastia L, Lee BA, Keller W, Proudfoot NJ. 1998. Coupling termination of transcription to messenger RNA maturation in yeast. Science 280: $298-301$.

Buratowski S. 2009. Progression through the RNA polymerase II CTD cycle. Mol Cell 36: 541-546.

Carswell S, Alwine JC. 1989. Efficiency of utilization of the simian virus 40 late polyadenylation site: Effects of upstream sequences. Mol Cell Biol 9: 4248-4258.

Chapman RD, Conrad M, Eick D. 2005. Role of the mammalian RNA polymerase II C-terminal domain (CTD) nonconsensus repeats in CTD stability and cell proliferation. Mol Cell Biol 25: 7665-7674.

Cope GA, Deshaies RJ. 2006. Targeted silencing of Jab1/Csn5 in human cells downregulates SCF activity through reduction of F-box protein levels. BMC Biochem 7: 1. doi: 10.1186/1471-2091-7-1.

Fong N, Bentley DL. 2001. Capping, splicing, and 3' processing are independently stimulated by RNA polymerase II: Different functions for different segments of the CTD. Genes Dev 15: 1783-1795.

Fong N, Bird G, Vigneron M, Bentley DL. 2003. A 10 residue motif at the C-terminus of the RNA pol II CTD is required for transcription, splicing, and 3' end processing. EMBO J 22: 4274-4282.

Fung TK, Siu WY, Yam CH, Lau A, Poon RY. 2002. Cyclin F is degraded during G2-M by mechanisms fundamentally different from other cyclins. J Biol Chem 277: 35140-35149.

Gerber HP, Hagmann M, Seipel K, Georgiev O, West MA, Litingtung Y, Schaffner W, Corden JL. 1995. RNA polymerase II C-terminal domain required for enhancer-driven transcription. Nature 374: 660-662.

Guialis A, Morrison KE, Ingles CJ. 1979. Regulated synthesis of RNA polymerase II polypeptides in Chinese hamster ovary cell lines. J Biol Chem 254: 4171-4176.

Hinkson IV, Elias JE. 2011. The dynamic state of protein turnover: It's about time. Trends Cell Biol 21: 293-303.

Kuehner JN, Pearson EL, Moore C. 2011. Unravelling the means to an end: RNA polymerase II transcription termination. Nat Rev Mol Cell Biol 12: 283-294.

Laurencikiene J, Kallman AM, Fong N, Bentley DL, Ohman M. 2006. RNA editing and alternative splicing: The importance of cotranscriptional coordination. EMBO Rep 7: 303-307.

Lee DH, Goldberg AL. 1998. Proteasome inhibitors: Valuable new tools for cell biologists. Trends Cell Biol 8: 397-403.

Martins SB, Rino J, Carvalho T, Carvalho C, Yoshida M, Klose JM, de Almeida SF, Carmo-Fonseca M. 2011. Spliceosome assembly is coupled to RNA polymerase II dynamics at the $3^{\prime}$ end of human genes. Nat Struct Mol Biol 18: 1115-1123.

Meininghaus M, Chapman RD, Horndasch M, Eick D. 2000. Conditional expression of RNA polymerase II in mammalian cells: Deletion of the carboxyl-terminal domain of the large subunit affects early steps in transcription. J Biol Chem 275: 24375-24382.

Nag A, Narsinh K, Kazerouninia A, Martinson HG. 2006. The conserved AAUAAA hexamer of the poly(A) signal can act alone to trigger a stable decrease in RNA polymerase II transcription velocity. RNA 12: 1534-1544.

Nag A, Narsinh K, Martinson HG. 2007. The poly(A)-dependent transcriptional pause is mediated by CPSF acting on the body of the polymerase. Nat Struct Mol Biol 14: 662-669.

Nguyen VT, Giannoni F, Dubois MF, Seo SJ, Vigneron M, Kedinger C, Bensaude O. 1996. In vivo degradation of RNA polymerase II largest subunit triggered by $\alpha$-amanitin. Nucleic Acids Res 24: 2924-2929.

Orozco IJ, Kim SJ, Martinson HG. 2002. The poly(A) signal, without the assistance of any downstream element, directs RNA polymerase II to pause in vivo and then to release stochastically from the template. J Biol Chem 277: 42899-42911.

Papavassiliou AG, Treier M, Chavrier C, Bohmann D. 1992. Targeted degradation of c-Fos, but not v-Fos, by a phosphorylation-dependent signal on c-Jun. Science 258: 1941-1944.

Park NJ, Tsao DC, Martinson HG. 2004. The two steps of poly(A)dependent termination, pausing and release, can be uncoupled by truncation of the RNA polymerase II CTD. Mol Cell Biol 24: 40924103.

Qu X, Perez-Canadillas JM, Agrawal S, De Baecke J, Cheng H, Varani G, Moore C. 2006. The C-terminal domains of vertebrate CstF-64 and its yeast orthologue Rna15 form a new structure critical for mRNA 3'-end processing. J Biol Chem 282: 2101-2115.

Richard P, Manley JL. 2009. Transcription termination by nuclear RNA polymerases. Genes Dev 23: 1247-1269.

Rosonina E, Blencowe BJ. 2004. Analysis of the requirement for RNA polymerase II CTD heptapeptide repeats in pre-mRNA splicing and $3^{\prime}$-end cleavage. RNA 10: 581-589.

Sims RJ III, Rojas LA, Beck D, Bonasio R, Schuller R, Drury WJ III, Eick D, Reinberg D. 2011. The C-terminal domain of RNA polymerase II is modified by site-specific methylation. Science 332: $99-103$.

Wagner EJ, Garcia-Blanco MA. 2002. RNAi-mediated PTB depletion leads to enhanced exon definition. Mol Cell 10: 943-949.

Whitelaw E, Proudfoot N. 1986. $\alpha$-thalassaemia caused by a poly(A) site mutation reveals that transcriptional termination is linked to $3^{\prime}$ end processing in the human $\alpha 2$ globin gene. EMBO J 5: 29152922.

Yamaguchi Y, Wada T, Watanabe D, Takagi T, Hasegawa J, Handa H. 1999. Structure and function of the human transcription elongation factor DSIF. J Biol Chem 274: 8085-8092. 

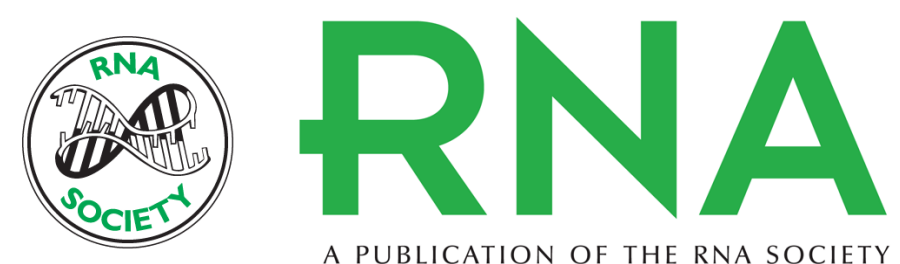

A PUBLICATION OF THE RNA SOCIETY

\title{
Prolonged $\alpha$-amanitin treatment of cells for studying mutated polymerases causes degradation of DSIF160 and other proteins
}

\author{
David C. Tsao, Noh Jin Park, Anita Nag, et al.
}

RNA 2012 18: 222-229 originally published online December 22, 2011

Access the most recent version at doi:10.1261/rna.030452.111

\section{References This article cites 32 articles, 17 of which can be accessed free at: http://rnajournal.cshlp.org/content/18/2/222.full.html\#ref-list-1}

\section{License}
Email Alerting Receive free email alerts when new articles cite this article - sign up in the box at the Service top right corner of the article or click here.

